\title{
Considerations of drug administration during breastfeeding
}

\author{
Ilinca TUDOSE-TRANULIS ${ }^{1}$, Anca Angela SIMIONESCU ${ }^{2,3}$, \\ Ana Maria Alexandra STANESCU ${ }^{4}$, Vlad DIMA 5 \\ ${ }^{1}$ Romanian Association of Lactation Consultants \\ ${ }^{2}$ Department of Obstetrics and Gynecology, \\ "Carol Davila" University of Medicine and Pharmacy, Bucharest, Romania \\ ${ }^{3}$ Department of Obstetrics and Gynecology, Filantropia Clinical Hospital, Bucharest, Romania \\ ${ }^{4}$ Department of Family Medicine, "Carol Davila“ University of Medicine and Pharmacy, Bucharest, Romania \\ ${ }^{5}$ Department of Neonatology, Filantropia Clinical Hospital, Bucharest, Romania
}

\begin{abstract}
Breastmilk is the best nutrition for infants. Unfortunately, almost all breastfeeding mothers require medication at some point during the breastfeeding period, which sometimes leads to the cessation of breastfeeding because of false beliefs about the true risk for the infant. Many prospects drugs information about side effects avoid any administration during pregnancy or in the postpartum period; also, because of fear, mothers choose to stop breastfeeding out, and general practitioners or pediatrist calculate lower doses to administrate. In this article, we want to draw attention to that even though there are no trials in neonates; we have much data on the safe use of medication during breastfeeding. In conclusion, we want to increase awareness about the necessity of increasing knowledge for mothers and healthcare personnel about indications, contraindications, and side effects of most used medication during breastfeeding.
\end{abstract}

Keywords: maternal milk, maternal breastfeeding, drugs

\section{INTRODUCTION}

Breastmilk is the best nutrition for infants [1], even for mothers with associated comorbidities [2]. Many studies have demonstrated that breastfeeding was associated with a reduction in the risk of baby overweight $[3,4]$, malocclusion, enteritis [5], increases in intelligence, and reduction of type 2 diabetes [4]. The actual recommendation is that all babies should be exclusively breastfed during the first 6 months of life, and breastfeeding should continue after the initiation of solid foods for two years or more, for as long as both mother and child desire [6] (WHO). More than $50 \%$ of postpartum women required at least one medicine [7].

Unfortunately, there are many concerns where breastfeeding is discontinued prematurely for various reasons, but misconceptions and false beliefs about drugs in breastmilk are the most founded. In a survey in the Netherlands, $11.5 \%$ of women discontinued breastfeeding because of medication, and much more chose to discontinue the treatment or not to take it at all [8]. Many prospects drugs information about side effects avoid any administration during pregnancy or in the postpartum period; also, because of fear, mothers 
choose to stop breastfeeding out, and general practitioners or pediatrist calculate lower doses to administrate.

The manufacturers' prescribing inserts discourage breastfeeding because of the risk of litigation, although there are studies that support their use during lactation for many drugs. Some doctors prefer interrupting breastfeeding for the duration of the treatment or ultimately, but mothers and babies suffer from this decision, which is unnecessary most of the time.

However, clinical trials on breastfeeding mothers are quite rare; less than 30 per cent of drugs have been studied [9-11]. In 2014, the FDA issued the Pregnancy and Lactation Labeling Rule requiring manufacturers to update drug labels with any information relevant to pregnant or breastfeeding women but did not require this information to be collected through clinical trials $[12,13]$. Due to COVID-19 pandemics, the U.S. Food and Drug Administration (FDA) have announced their commitment to work with other regulators and industry to support the inclusion of pregnant and lactating women in clinical research worldwide [14].

In this article, we want to draw attention to that although there are no trials in neonates, we have much data on the safe use of medication during breastfeeding.

\section{EVIDENCE ABOUT BREASTMILK PASSAGE AND MOTHER DRUG EXPOSURE}

Existing kinetic modelling systems do not provide an accurate result; we still cannot tell the exact level of the medicine transfers through milk to the baby $[11,13]$.

Drugs transfer into breastmilk depends on their physicochemical characteristics: weight, lipid solubility, protein binding, acid-base dissociation constant ( $p K a)$ $[15,16]$.

We summarize for the main drugs potentially administered during postpartum the main findings, as follow [11,15-22]:

- Medication in plasma passes into milk mainly by diffusion, only after attaining a certain plasma level.

- Drugs with low bioavailability do not attain a high plasma level (e.g., morphine, heparins, omeprazole, vancomycin);

- Medication concentrated in the gastrointestinal tract of the infants can cause adverse effects like diarrhoea (e.g., antibiotics)

- Plasma levels of the drug are in equilibrium with milk level of the drug; the drug passes back into the plasma when plasma levels drop

- Drugs that are weak bases may become trapped in breastmilk (e.g., barbiturates, ranitidine)

- During the first 4-6 days postpartum, a larger quantity of the drug can pass into the milk, but the amount of colostrum for the first 1-2 days is low

- Most drugs should dissolve through the bilayer lipid membranes of the alveolar cells in order to pass into breastmilk.

- Lipid soluble drugs pass into breastmilk easier, but they also have high volumes of distribution, so usually it means low levels in milk (e.g., diazepam, chlorpromazine)

- Drugs active in the central nervous system pass easier into milk

- Drugs with high molecular weight (over 800 Daltons) pass less into breastmilk (e.g., heparin, insulin, interferon - very large molecular size, they virtually do not pass into milk); drugs with low molecular weight (e.g., lithium, psychotropic drugs) transfers easily into breastmilk.

- Drugs with high protein binding pass less into milk (e.g., nonsteroidal anti-inflammatory drugs, warfarin)

- The shorter the half-life of a drug, the lower the milk level

- The milk-to-plasma ratio of a drug is relevant when we know the plasma level; a low M/P ratio means little drug in breastmilk, but high $\mathrm{M} / \mathrm{P}$ does not necessarily mean a high drug level in milk (e.g., ranitidine)

- The relative infant dose is important in evaluating the safety of a drug, but we need to know the concentration of the drug in the milk, the quantity of transferred milk, and dose for infants; in term infants, relative infant dose under $10 \%$ of mother's dose is considered safe.

\section{MATERNAL FACTORS CHARACTERISTICS RELATED TO MILK PRODUCTION AND MEDICATION TRANSFER}

Maternal factors that affect drug transfer and that need to be taken into consideration when treating the mother are quantity of milk, maternal dose, half-life, way of administration of the drug. Maternal milk production related to the age of infant is:

- Days 2-4 postpartum - minimal dose because the low quantity of colostrum

- Infant 2 weeks - 6 months - high volume of milk

- After 12 months of age - lower quantities of milk.

Many authors demonstrate that the older the infant, the better the metabolization and excretion of medication [11,23]. A high maternal dose of drug administration was correlated with higher levels in breastmilk. Also, the longer the release of the drug, the higher the levels in breastmilk. Therefore, drugs with a short half-life should be taken a right after breastfeeding 
[11]. Topical drugs usually do not pass into breastmilk (e.g., steroids, retinoids, antibiotics used on small areas of skin, inhaled asthma drugs virtually do not pass into the milk, local anaesthetics like dental procedures) [24-26].

\section{CONCLUSIONS}

Physicians should make their best choice of necessary drugs to sustain and encourage breastfeeding, the best nutrition for infants. All infants should be evaluated clinically because some aspects of the child's status can influence the risk of the medication, for example, prematurity, infants with comorbidities or duration of maternal treatment.

\section{REFERENCES}

1. Błachnio M, Dmoch-Gajzlerska $E$, Bednarczyk M. The influence of structured prenatal education on the use of specialist lactation counselling in case of difficulties. J Mother Child. 2021;23(4):216-226.

2. Matsunaga $M$, Kataoka $Y$, Igarashi $Y$, et al. Breastfeeding support and barriers to women with gestational diabetes mellitus: a nationwide cross-sectional survey of hospitals in Japan. BMC Pregnancy Childbirth. 2021;21(1):555.

3. Yan J, Liu L, Zhu Y, Huang G, et al. The association between breastfeeding and childhood obesity: a meta-analysis. BMC Public Health. 2014;14:1267.

4. Victora CG, Bahl R, Barros AJD, et al. Breastfeeding in the 21st century: epidemiology, mechanisms, and lifelong effect. Lancet. 2016;378:475-490.

5. Prell C, Koletzko B. Breastfeeding and Complementary Feeding. Dtsch Arztebl Int. 2016 Jun 24;113(25):435-44.

6. Breastfeeding-WHO|World Health Organization. Available at: https://www.who. int/health-topics/breastfeeding

7. Saha MR, Ryan K, Amir LH. Postpartum women's use of medicines and breastfeeding practices: a systematic review. Int Breastfeed J. 2015 Oct 28;10:28.

8. Schirm E, Schwagermann M, Tobi $\mathrm{H}$, et al. Drug use during breastfeeding. A survey from the Netherlands. Eur J Clin Nutr. 2004;58:386-390.

9. Wambach K, Spencer B. Breastfeeding and Human Lactation, 6th edition. Jones \& Bartlett Learning, 2019.

10. Hetzel Campbell S, Lauwers J, Mannel R, Spencer B. Core Curriculum for
Interdisciplinary Lactation Care, 1st edition. Jones \& Bartlett Learning, 2018.

11. Lawrence RA, Lawrence RM. Breastfeeding - A Guide for the Medical Profession, 9th edition. Elsevier, 2014.

12. Pregnancy and Lactation Labeling (Drugs) Final Rule 2014 FDA administration. Available at: https://www.fda.gov/drugs/ labeling-information-drug-products/ pregnancy-and-lactation-labeling-drugs-finalrule.

13. Pernia S, DeMaagd G. The New Pregnancy and Lactation Labeling Rule. P T. 2016 Nov;41(11):713-715.

14. Global Regulators Envision Paradigm Shift Toward Inclusion of Pregnant and Breastfeeding Women in Clinical Research for Medicines and Vaccines. Available at: https://www.fda.gov/news-events/fda-voices/ global-regulators-envision-paradigm-shifttoward-inclusion-pregnant-andbreastfeeding-women-clinical.

15. Hale TW, Hartmann PE. Textbook of Human Lactation. Springer, 2007.

16. Rowe H, Baker T, Hale TW. Maternal medication, drug use, and breastfeeding. Pediatr Clin North Am. 2013;60(1):275-94.

17. Fleishaker JC, Desai N, McNamara PJ. Factors affecting the milk-to-plasma drug concentration ratio in lactating women: physical interactions with protein and fat. J Pharm Sci. 1987;76(3):189-93.

18. Daei M, Khalili H, Heidari Z. Direct oral anticoagulant safety during breastfeeding: a narrative review. Eur J Clin Pharmacol. 2021 May 8.

19. Zhao Y, Arya R, Couchman L, et al. Are apixaban and rivaroxaban distributed into
Conflict of interest: none declared Financial support: none declared human breast milk to clinically relevant concentrations? Blood. 2020; 136(15):1783-1785.

20. He L, Yang L, Zhang H, Luo Q. Efficacy and safety of interferon on neonates with respiratory syncytial virus pneumonia. Exp Ther Med. 2020;20(6):220.

21. Behpour M, Nojavan S, Asadi S, et al. Combination of gel-electromembrane extraction with switchable hydrophilicity solvent-based homogeneous liquid-liquid microextraction followed by gas chromatography for the extraction and determination of antidepressants in human serum, breast milk and wastewater. J Chromatogr A. 2020;1621:461041.

22. Toker SE, Kızılçay GE, Sagirli O. Determination of levofloxacin by HPLC with fluorescence detection in human breast milk. Bioanalysis. 2021;13(13):1063-1070.

23. Nimbalkar S, Vasa RK. Changes in preterm breast milk composition with advancing infant age. Indian Pediatr. 2014 Dec;51(12):966-7.

24. Moore CB, Bond JD, Bundoc EG, Hefley JB, Wofford KA, Bonds RL. Resuming Breastfeeding After Surgery: Influencing Practice Recommendations. J Perianesth Nurs. 2021 May 6:S1089-9472(20)30408-1.

25. Mitchell J, Jones W, Winkley E, et al. Guideline on anaesthesia and sedation in breastfeeding women 2020: Guideline from the Association of Anaesthetists.

Anaesthesia. 2020 Nov;75(11):1482-1493.

26. Simionescu AA, Danciu BM, Stanescu AMA. State-of-the-Art Review of PregnancyRelated Psoriasis. Medicina. 2021;57(8):804. 